\title{
CPRM: Cooperative Position based Routing scheme for MANETs
}

\author{
Ginumol Jose ${ }^{1}$, Prof. Kuttyamma A J ${ }^{2}$ \\ ${ }^{I}$ (Department of IT, Rajagiri School of Engineering \& Technology, Kerala, India) \\ ${ }^{2}$ (Department of IT, Rajagiri School of Engineering \& Technology, Kerala, India)
}

\begin{abstract}
A mobile ad hoc network is a wireless communication network, where nodes that are not within direct transmission range of each other will require other nodes to forward data. Routing is difficult in MANET because mobility may cause radio links to break frequently. Mobile nodes keep changing their position in an unpredictable manner. A routing algorithm must keep track of the destination's position and modify routes whenever the old routes become stale. In a high mobility MANET, although every mode can move freely, they usually follow some mobility patterns. Fast changes of topology increases the complexity of routing. Due to mobility of the nodes to another location, some of the data packets will be missed and does not deliver the data packets to the destination in a reliable manner. This project proposes a Cooperative Position based Routing scheme for mobile ad hoc networks (CPRM). In this, the routing is carried out based on the positions of nodes in the network. The main objective of this project is to achieve the reliable data delivery in the network. In this scheme, the source node finds a forwarder node from its neighbor list, for transmitting data. The forwarder in turn finds the next forwarder and this process continues until the destination is reached. Performance of the proposed system is compared with that of AODV using Network Simulator.
\end{abstract}

Keywords: Cooperative routing, Forwarder, Position-based routing.

\section{Introduction}

A mobile ad hoc network is a wireless communication network, where nodes that are not within direct transmission range of each other will require other nodes to forward data. It can operate without existing infrastructure, supports mobile users, and falls under the general scope of multi hop wireless networking. Such a networking paradigm was originated from the needs in battlefield communications, emergence operations, search and rescue, and disaster relief operations. Later, it found civilian applications such as community networks. A great deal of research results have been published since its early days in the 1980s. The most salient research challenges in this area include end-to-end data transfer, link access control, security, and providing support for real-time multimedia streaming.

Routing protocols in mobile ad hoc networks can be categorized using an array of criteria. The most fundamental difference among these protocols is the timing of routing information exchange. On one hand, a protocol may require that nodes in the network should maintain valid routes to all destinations all the time. In this case, the protocol is considered to be proactive, ie, table driven. Examples of proactive routing protocols include Destination-Sequenced Distance Vector (DSDV) and Optimized Link State Routing (OLSR). On the other hand, if nodes in the network do not always maintain routing information, when a node receives data from the upper layer for a given destination, it must first find out how to reach the destination. This approach is called reactive, ie, on demand. Dynamic Source Routing (DSR) and Ad hoc On Demand Distance Vector (AODV) fall in this category.

Routing is difficult in MANET because mobility may cause radio links to break frequently. Mobile nodes keep changing their position in an unpredictable manner. A routing algorithm must keep track of the destination's position and modify routes whenever the old routes become stale. In a high mobility MANET, although every mode can move freely, they usually follow some mobility patterns. Fast changes of topology increases the complexity of routing. Location information of each mobile node should be important for routing. It may also cause routes to be broken more often, and re-routing becomes more frequent. It becomes a time consuming process if the communicating route fails frequently. So the proposed system supports the reliable packet delivery to the destination using a set of forwarder nodes.

Position based routing algorithms eliminate some of the limitations of topology- based routing by using additional information. They require that information about the physical position of the participating nodes be available. Commonly each node determines its own position through the use of some type of positioning service. A location service is used by the sender of a packet to determine the position of the destination and include it in the packet's destination. The routing decision at each node is then based on the destination's position contained in the packet and the position of the forwarding node's neighbors. Position based routing thus does not require the establishment or maintenance of routes. The nodes have neither to store routing tables nor to transmit messages to keep routing tables up to date. As a further advantage, position-based routing supports the delivery of packets to all nodes in a given geographic region in a natural way. This type of service is called 
geocasting. This paper proposes the aggressive use of geography to achieve the cooperative communication in wireless network.

Contributions of the systematic solution proposed in this paper are highlighted as follows.

- The underlying routing protocol is AODV. Changes are made to the routing of AODV based on the position of nodes in the network. A list of forwarders is selected based on their distance to the destination and the data packets are routed through these forwarder nodes.

- When a flow of data packets are forwarded towards their destination, the route can be adjusted by intermediate forwarders based on the changes in the topology.

The rest of this article is organized as follows. Section II reviews related work on routing schemes for mobile ad hoc networks, with special focus on CORMAN and AODV. Section III describes the basic idea of CPRM. Section IV deal with the performance evaluation of CPRM. We use computer simulation to test the performance of CPRM, and the settings and results of these experiments are in Section IV. Section V concludes the article with an outlook to future research.

\section{Related Work}

A network route is simply a path between a source and destination. Routing is the process of finding a valid path between two nodes and utilizing the path for data communications. MANET routing faces significantly more challenges than fixed (or wired) networks. One challenge is securing broadcast wireless communication in an un-trusted environment; another challenge is finding the route itself. Routing is further challenged in mobile ad hoc networks since network topology is not static. Node movement creates a continually changing network topology that MANET protocols must consider. Addition- ally, a node must rely on its neighbors (those within its wireless transmission distance) for network topology information and to delay data to nodes outside its transmission distance. Ad hoc routing protocols are commonly classified according to their routing strategy as reactive (sometimes referred to as source-initiated or on-demand) or proactive (sometimes referred to as table-driven)[10].

In paper [1], a Cooperative opportunistic routing scheme for MANETs is described. CORMAN is Cooperative Opportunistic Routing scheme for MANets. CORMAN forwards data in a batch-operated fashion. A flow of data packets are divided into batches. All packets in the same batch carry the same forwarder list when they leave the source node. In CORMAN, the underlying routing protocol used is, Proactive Source Routing (PSR), which provides each node the complete routing information to all other nodes in the network. Thus, the forwarder list contains the identities of the nodes on the path from the source node to the destination. As packets progress in the network, the nodes listed as forwarders can modify the forwarder list if any changes have been observed in the network topology. This is referred to as large-scale live update in our work. In addition, this also allows some other nodes that are not listed as forwarders to retransmit data if this turns out to be helpful, referred to as small-scale retransmission.

PSR [3] provides every node with a Breadth First Spanning Tree (BFST) of the entire network rooted at it. To do that, nodes periodically broadcast the tree structure to its best knowledge in each of the iteration. Based on the information collected from neighbors during the most recent iteration, a node can expand and refresh its knowledge about the network topology by constructing a deeper and more recent BFST. This knowledge will be distributed to its neighbors in the next round of operation.

CORMAN has some disadvantages. It uses proactive routing protocol, so need to maintain the complete routes every time. It broadcasts the packets, so it increases the network traffic. Also, the storage requirements are more, since the routing table contains more entries.

Ad hoc On Demand Distance Vector (AODV) is a reactive protocol that creates and maintains routes only when they are requested. On a given node, the routing table stores only information about the next hop to the desired destination and a sequence number received from the destination, preserving the freshness of the information stored. Literature [10] contains the details of AODV.

\section{Cprm: Cooperative Position-Based Routing Scheme For Manets}

The main proposal of the cooperative communication is to ensure better performance in the core of the MANET network. A CPRM contains the following mechanisms

- $\quad$ Neighbor discovery

- Discovery of forwarder node from the neighbor list

- $\quad$ Position based routing

The source finds its neighbors and from the neighbor list it finds a forwarder node. The routing is carried out through these forwarder nodes. When data packets are received by and stored at a forwarding node, the node may have a different view of how to forward them to the destination. Since this node is closer to the 
destination than the source node. This means that the forwarding node has more updated routing information. In AODV, each node has information about the next hop node to the destination and the sequence number from the destination. The sequence number is for ensuring the freshness of the information stored at each node. In my project CPRM, there is no need to establish and maintain the route by sending the route request and route reply packets. The forwarder node is selected based on the distance to the destination. The node having the smallest distance to the destination, among all the neighbors of the node, is selected as the forwarder node.

\section{A. Selection of Neighbors}

A MANET (mobile ad hoc network) is a collection of mobile nodes that cooperate to forward packets for each other to allow them to communicate beyond direct wireless transmission range. The source node has updated knowledge of the network structure within its proximity but its knowledge about further areas of the network can be obsolete due to node mobility. This error becomes worse as the data packet is forwarded towards the destination node. For finding the neighbors, first calculate the distance of every node to every other node. Then a value is fixed as the range of the node. The nodes, that comes under the range of a node is considered as the neighbors of that node. When the topology change occurs, the neighbors of the node are also changes. The nodes that are the neighbors of a particular node at a time may not the neighbors of that node at a later time.

\section{B. Selection of Forwarding nodes}

When considering a mobile ad hoc network, each node has updated knowledge of the network structure within its proximity but its knowledge about further areas of the network can be obsolete due to node mobility. This error becomes worse as the data packet is forwarded towards the destination node. To address this issue, intermediate nodes should have the ability to update the route adaptively with their new knowledge when forwarding data packet. The forwarding router decides only information about a source or routers immediate neighbors in the network topology. For routing of data packets, selection of forwarder nodes is essential. Source first finds its neighbors and from the neighbor list it selects the forwarder node. The selection of the forwarder node is based on the distance to the destination. For this, the distance of each neighbor to the destination is calculated. The node having the minimum distance to the destination is selected as the forwarder node.

\section{Position based Routing}

The Position based routing for wireless networks uses the positions of routers and a packets destination to make packet forwarding decisions. It makes forwarding decisions using only information about a router's immediate neighbors in the network topology. By keeping state only about the local topology, it scales better in per-router state than shortest path. Due to node's movement, some forwarding candidates may fail to receive the packets. After finding the forwarder node, the source sends packets through this forwarder node.

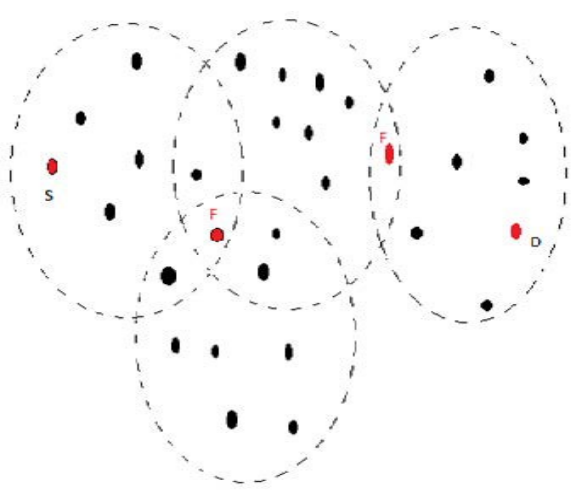

Fig.1 position based routing

Then the forwarder node finds the next forwarder node and sends packet to it. This process continues until the destination is reached. When topology changes due to node mobility, it is the responsibility of the forwarder node, having the packet now, to find the next hop to the destination. The source need not reconstruct the route, when an old route became stale. So in CPRM, the routing is done through a set of forwarder nodes.

In fig.1, the routing from source to destination is done through two forwarders. The source first found a forwarder and send data packets to it. That forwarder node in turn finds the next forwarder node for packet transmission. In this figure, the path to the destination is S-F-F-D. When topology changes due to mobility of nodes, the path to the destination also changes. Because of the forwarder nodes for packet transmission changes when topology change occurs. 
D. Merits of Proposed system

- Does not require route establishment by sending route request packet

- $\quad$ No need to maintain the route information

- $\quad$ More reliable data transfer

- $\quad$ Packet drop is less

- $\quad$ Packet delivery ratio is high

- Throughput is high

\section{Performance Evaluation}

In this section, we study the performance of CPRM by running computer simulation using Network Simulator ns-2 (version 2.35). Different factors are considered and X-graphs are plotted based on the comparison. The performance of the CPRM is compared with that of AODV by considering the following factors

$\begin{array}{ll}- & \text { Throughput } \\ \text { - } & \text { Packet Delivery ratio } \\ \text { A. } & \text { Experimentized overhead } \\ & \end{array}$

We compare the performance of CPRM with that of AODV [10]. We select AODV as the baseline because AODV is a widely adopted routing protocol in MANETs, and its behavior both in ns-2 and real network operation is well understood by the research community. In modeling node motion, we adopt the random waypoint model to generate the simulation scenarios. In this model, each node moves towards a series of target positions. Once it has reached a target position, it may pause for a specific amount of time before moving towards the next position. We inject CBR (constant bit rate) data flows in the network, which are carried by UDP. The simulation model is given in the fig.2.

\begin{tabular}{|l|l|}
\hline SIMULATOR & Network Simulator 2 \\
\hline NUMBER OF NODES & Random \\
\hline TOPOLOGY & Random \\
\hline INTERFACE TYPE & Phy/WirelessPhy \\
\hline MAC TYPE & 802.11 \\
\hline QUEUE TYPE & Droptail/Priority Queue \\
\hline QUEUE LENGTH & 200 Packets \\
\hline ANTENNA TYPE & Omni Antenna \\
\hline PROPAGATION TYPE & Two ray Ground \\
\hline TRANSPORT AGENT & TCP \\
\hline APPLICATION AGENT & FTP \\
\hline SIMULATION TIME & 50 seconds \\
\hline
\end{tabular}

Fig.2 simulation model

PDR (Packet Delivery Ratio) is the proportion to the total amount of packets reached the receiver and amount of packet sent by source. If the amount of malicious node increases, PDR decreases. The higher mobility of nodes causes PDR to decrease. As the node density decreases, this rate gradually goes down to about $60 \%$. In contrast, AODV's PDR ranges between $60 \%$ and $80 \%$ for dense networks. There are two reasons for the PDR penalty for AODV to operate in sparse networks. First, data packets are forwarded using traditional IP forwarding in AODV. When channel quality varies, a packet may be lost at the link layer. After a few failed retransmits, it will be dropped by the network layer. CPRM facilitates cooperative data forwarding using the link quality diversity at different receivers. Consequently, CPRM has a strong resilience to link quality fluctuation and node mobility. Second, the route search of AODV does not function well with unreliable links. Recall that, in AODV, when a node finds that it does not have a next hop available for a given data packet, it broadcasts a RREQ (route request) to find one. Both the destination and any intermediate node that has a valid cached route can reply with a RREP. When links were perfectly symmetric, the RREP packet would take the inverse path leading to the initiating node of the route search. However, when links suffer from fading, the RREP packets may not be able to propagate back to the initiator because of transient low link quality in the reverse direction. As a result, it takes AODV much longer to obtain stable routes.

CPRM uses position-based routing. It makes forwarding decisions using only information about a router's immediate neighbors in the network topology. By keeping state only about the local topology, it scales better in per-router state than shortest-path. Due to node's movement, some forwarding candidates may fail to receive the packets. After finding the forwarder node, the source sends packets through this forwarder node. 
Then the forwarder node finds the next forwarder node and sends packet to it. This process continues until the destination is reached. When topology changes due to node mobility, it is the responsibility of the forwarder node, having the packet now, to find the next hop to the destination. The source need not reconstruct the route, when an old route became stale. Fig. 3 shows the X-graph based on the comparison of PDR of both CPRM and AODV. The figure shows that the packet delivery ratio of CPRM is higher when compared to that of AODV.

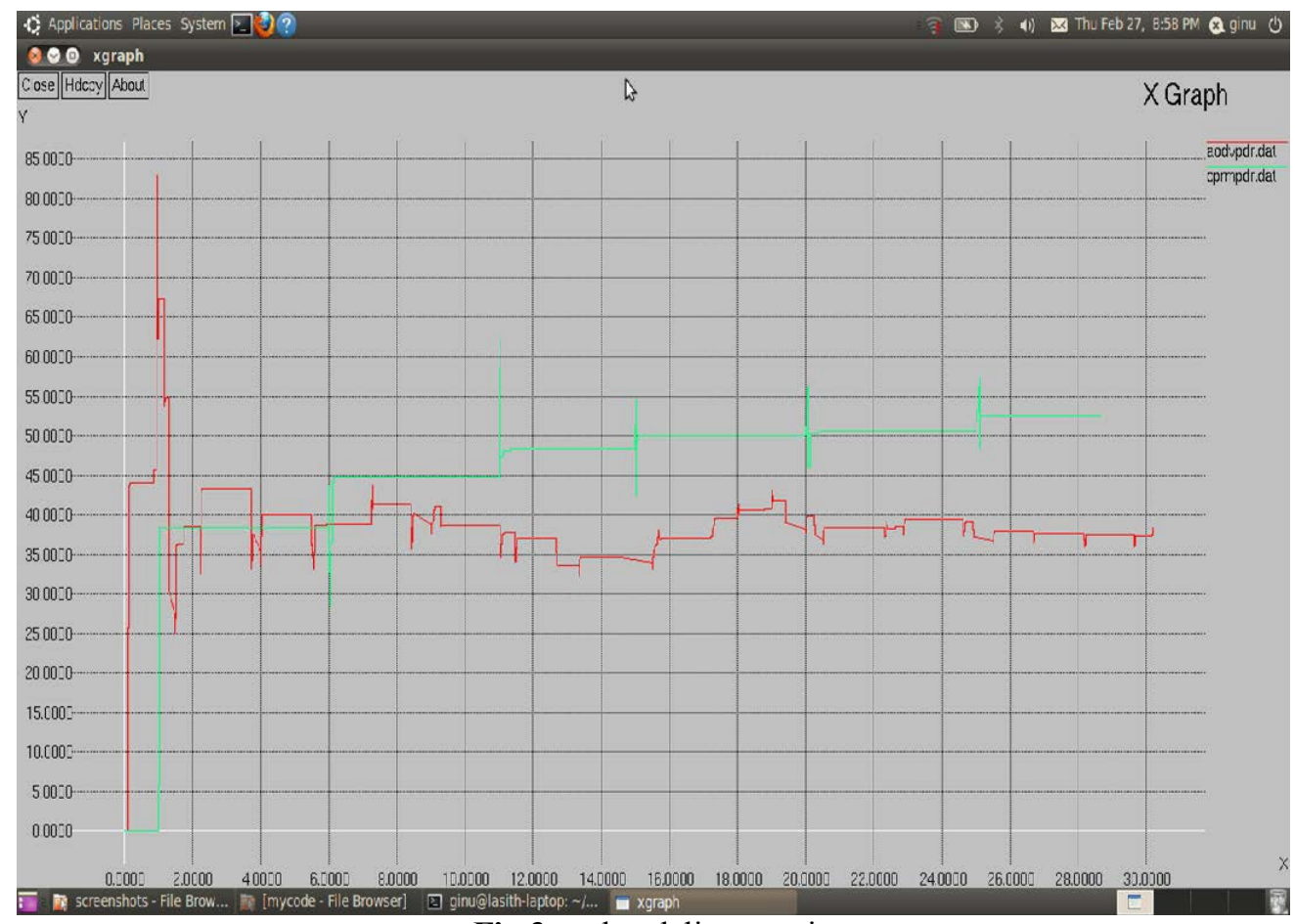

Fig.3 packet delivery ratio

For evaluation of the performance of the proposed system, throughput for both AODV and CPRM is also calculated. Throughput is the amount of data delivered per unit time. The X-graph for the throughput for both AODV and CPRM is given in fig.4. From the figure, it is understood that the throughput of CPRM is higher when compared with that of AODV.

Normalized overhead is the overhead that is incurred while routing a packet. It is also considered for comparing the performance of CPRM with that of AODV. Fig.5 shows the X-graph based on the comparison of NOH of both CPRM and AODV. The figure shows that the normalized overhead of both CPRM and AODV is almost same.

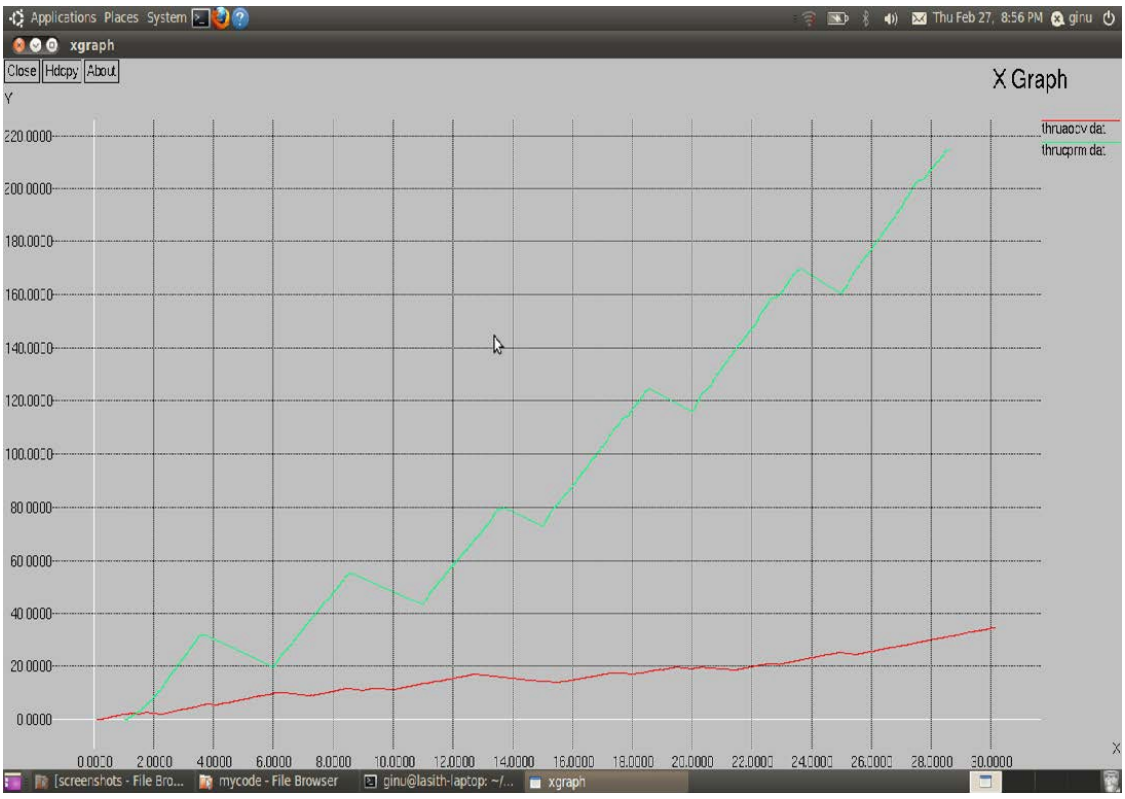

Fig.4 throughput 


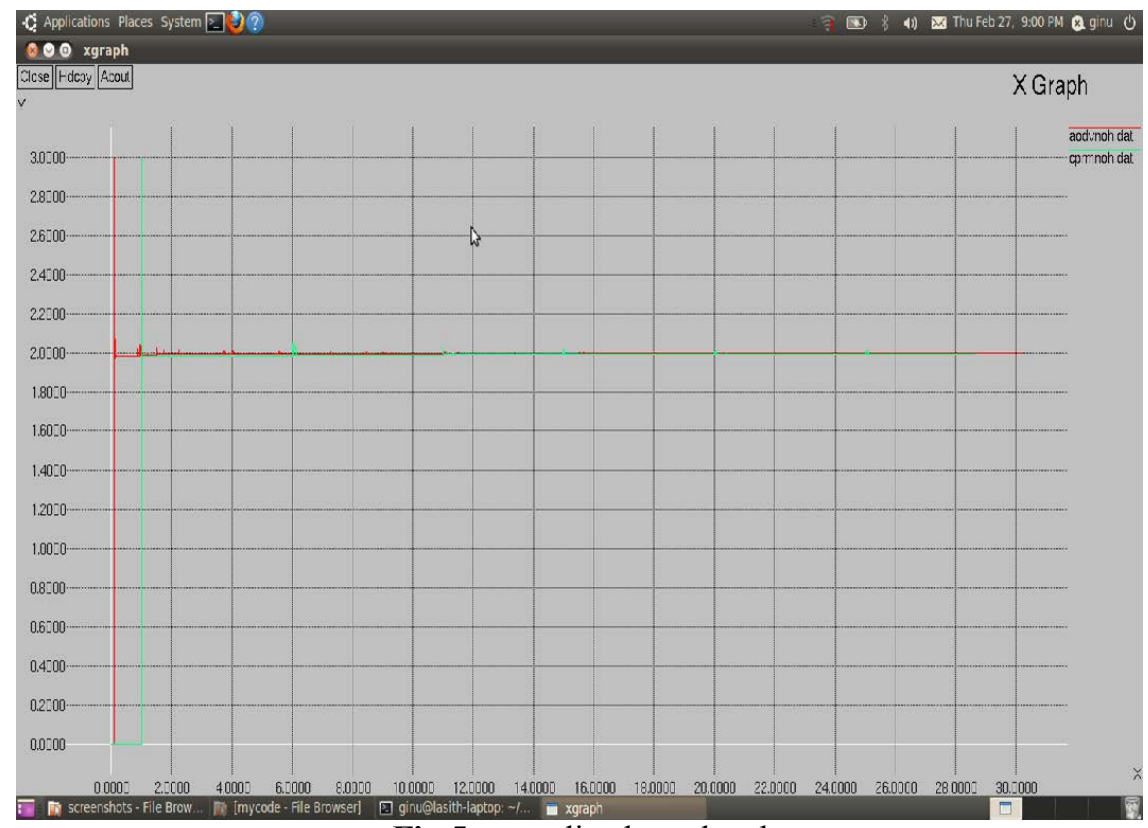

Fig.5 normalized overhead

\section{Conclusion And Future Enhancement}

Routing is difficult in MANET because mobility may cause radio links to break frequently. Mobile nodes keep changing their position in an unpredictable manner. A routing algorithm must keep track of the destination's position and modify routes whenever the old routes become stale. In a high mobility MANET, although every mode can move freely, they usually follow some mobility patterns. Fast changes of topology increases the complexity of routing. In this project, problem of reliable data delivery in mobile ad hoc networks is considered. This paper proposes a cooperative position based routing scheme for mobile ad hoc networks. Due to node's movement, some forwarding candidates may fail to receive the packets. After finding the forwarder node, the source sends packets through this forwarder node. Then the forwarder node finds the next forwarder node and sends packet to it. This process continues until the destination is reached. When topology changes due to node mobility, it is the responsibility of the forwarder node, having the packet now, to find the next hop to the destination. The source need not reconstruct the route when an old route became stale. In this, forwarding nodes are selected based on the distance to the destination, and routing is carried out through these forwarder nodes. In case of link failure, the forwarding node will find the next node to the destination. The performance of the proposed system is evaluated by comparing it with AODV. It shows CPRM is more efficient than AODV.

CPRM can be extended in the following ways:

- $\quad$ Selection of re-transmitters :

Some re-transmitter nodes are selected for transmitting the missed packets. Only the nodes located in the forwarding area would get the chance to be re-transmitters (backup) nodes. The forwarding area is determined by the sender and the next hop node. A node located in the forwarding area satisfies the following two conditions:

- It makes positive progress toward the destination

- Its distance to the next hop node should not exceed half of the transmission range of a wireless node, so that ideally all the re-transmitters candidates can hear from one another.

Next hop node (best forwarder) only forwards the packet to destination. If the best forwarder does not forward the packet in certain time slots, suboptimal candidates will take turn to forward the packet according to a locally formed order. In cooperative position based routing, a source node sends the packet to the retransmitter's (backup) nodes and next hop. Then next hop again to find out the hops to forward the packet. After finding the next hop node A sends its data to back up nodes and next forwarding nodes. That information is overheard by previous back up nodes also since they are within the half of the communication range of next forwarding nodes. A packet with the same ID is received again by the previous back up nodes, it is discarded.

- $\quad$ Mobility Based Forwarding Node Selection : 
In CPRM, the forwarding nodes are selected based on the distance to the destination. In Mobile Ad hoc Networks if forwarding nodes have high mobility, may chances to make local topology inaccuracy. If the node involved in the forwarding path node moves frequently then there is the situation of frequent beacon update is required which leads to network traffic in turn packet drops. Hence it is required to select the nodes with low mobility which means selection of stable node as forwarder based on its mobility. The system with low mobility based forwarding node selection improves routing performance. Source node finds the distance of each neighbor from itself at particular time $(\mathrm{t})$. After certain time $(\mathrm{t}+\mathrm{T})$ it finds the distance again. If the difference between the two distances is less than the threshold, the neighbor is considered as highly stable neighbor. To apply highly stable forwarding distance between destination and highly stable neighbors are calculated. The neighbor which is having the minimum distance is selected as forwarder. Fig. 6 shows the diagram including mobility based forwarder nodes.

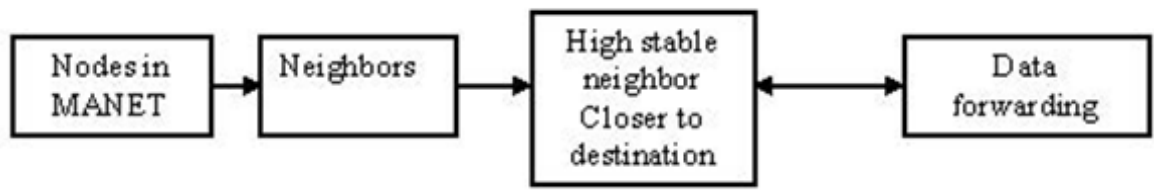

Fig.6 mobility based forwarder nodes

\section{References}

[1]. Zehua Wang, Yuanzhu Chen, Cheng Li,CORMAN: A Novel Cooperative Opportunistic Routing Scheme in Mobile Ad HocNetworks, IEEE Journal on selected areas in communications, vol. 30, no. 2, february 2012.

[2]. S. Biswas and R. Morris,ExOR: Opportunistic Multi-Hop Routing for Wireless Networks, in Proc. ACM Conference of the Special Interest Group on Data Communication (SIGCOMM), Philadelphia, PA, USA, August 2005, pp. 133144.

[3]. Z. Wang, C. Li, and Y. Chen,PSR: Proactive Source Routing Ad Hoc Networks, in Proc. 2011 IEEE Conference on Global Telecommunications (GLOBECOM), Houston, TX USA, December 2011.

[4]. Yang, F. Zhong, C. K. Yeo, B. S. Lee, and J. Boleng,Position Based Opportunistic Routing for Robust Data Delivery in MANETs, in Proc. 2009 IEEE Conference on Global Telecommunications (GLOBECOM), Honolulu, Hawaii, USA, December 2009, pp. 13251330 .

[5]. S. Murthy and J. J. Garcia-Luna-Aceves,An Efficient Routing for Wireless Networks, Mobile Networks and Applications, vol. 1, no. 2,pp. 183197 , October 1996.

[6]. S. Chachulski, M. Jennings, S. Katti, and D. Katabi,Trading Structure for Random- ness in Wireless Opportunistic Routing, in Proc. ACM Conference of the Special Interest Group on Data Communication (SIGCOMM), Kyoto, Japan, August 2007, pp. 169180.

[7]. M. K. Marina and S. R. Das,Routing Performance in the Presence of Unidirectional Links in Multihop Wireless Networks, in The Third ACM International Symposium on Mobile Ad Hoc Networking and Computing (MobiHoc02), Lausanne, Switzerland, June 2002 , pp. 1223.

[8]. I. Chlamtac, M. Conti, and J.-N. Liu,Mobile Ad hoc Networking: Imperatives and Challenges, Ad Hoc Networks, vol. 1, no. 1, pp. 1364, July 2003

[9]. D. Son, A. Helmy, and B. Krishnamachari,The Effect of Mobility Induced Location Errors on Geographic Routing in MobileAd Hoc Sensor Networks: Analysis and Improvement Using Mobility Prediction, IEEE Trans. Mobile Computing, vol. 3, no. 3, pp. 233- 245, July/Aug. 2004.

[10]. E. Royer,A review of current routing protocols for adhoc mobile wireless networks, IEEE PersonalCommunications, pp. 46-55, Apr. 1999.

[11]. D. Johnson, Y. Hu and D. Maltz,The Dynamic Source Routing Protocol (DSR) for Mobile Ad Hoc Networks for IPv4, RFC4728, February 2007. 